\title{
Post-LASIK Keratoectasia Developing with Sleep Apnea and Floppy Eyelid Syndrome: Case Report and Review of the Literature
}

\author{
${ }^{1}$ Noa Avni-Zauberman, ${ }^{2}$ David S Rootman
}

\begin{abstract}
We report a case of unilateral keratoectasia in a patient who underwent uneventful hyperopic LASIK 8 years prior and was diagnosed with obstructive sleep apnea syndrome (OSAS) and Floppy eyelid syndrome. The before and after LASIK topographies demonstrated clear development of the ectasia after the patient developed OSAS. The patient was treated with collagen crosslinking in the involved eye to stop ectatic progression. Our report emphasizes the importance of inquiring about the patient's sleeping postures and habits (specifically asking about OSAS) and a careful examination of eyelid laxity prior to refractive laser treatment.
\end{abstract}

Keywords: Post-LASIK ectasia, Floppy eyelid syndrome, Obstructive sleep apnea syndrome.

How to cite this article: Zauberman NA, Rootman DS. PostLASIK Keratoectasia Developing with Sleep Apnea and Floppy Eyelid Syndrome: Case Report and Review of the Literature. Int J Kerat Ect Cor Dis 2014;3(1):44-46.

\section{Source of support: Nil}

Conflict of interest: None

\section{INTRODUCTION}

Floppy eyelid syndrome (FES) was first described by Culbertson and Ostler in 1981. ${ }^{1}$ This condition is characterized by very elastic upper lids that became easily distorted with minimal lateral traction. This syndrome was found to be associated with systemic and ocular conditions including dermatochalasis, ${ }^{1}$ blepharitis ${ }^{2-5}$ and blepharoptosis. ${ }^{3,6}$ Superficial punctate keratopaty, ${ }^{5}$ scarring and neovascularization, ${ }^{1,7}$ microbial keratitis ${ }^{5-7}$ and even corneal perforation ${ }^{5,8}$ have also been reported. These corneal manifestations are most probably the result of mechanical eyelid rubbing and chronic exposure keratopathy during sleep. FES was found to be associated also with keratoconous both clinical ${ }^{5,9}$ and subclinical (about $\left.20 \%\right)^{5}$

\footnotetext{
${ }^{1}$ Clinical Fellow, ${ }^{2}$ Professor

1,2Department of Ophthalmology and Vision Sciences, Yonge Eglinton Laser Center, University of Toronto, Ontario, Canada

Corresponding Author: Noa Avni-Zauberman, Clinical Fellow Department of Ophthalmology and Vision Sciences, Yonge Eglinton Laser Center, University of Toronto, Ontario, Canada Phone: 16479698981, e-mail: avnin.z@gmail.com
}

One of the most consistently systemic reported associations of FES is that of obstructive sleep apnea syndrome (OSAS) ${ }^{3,10}$ Sleep apnea syndrome is characterized by recurrent complete or partial upper airway obstructions during sleep. In addition, OSAS has been suggested as a possible cause for FES, and this hypothesis is strengthened by a report of FES resolving simply with the use of a continuous positive airway pressure mask (CPAP). ${ }^{11}$

We report a case of unilateral keratoectasia in a patient who underwent uneventful hyperopic LASIK 8 years prior and was diagnosed with OSAS and FES.

\section{CASE REPORT}

A 55-year-old male was referred to our refractive center 9 years ago for refractive laser vision correction. His medical history was unremarkable, excluding sporadic bronchitis and overweight.

On examination, his uncorrected visual acuity (UCVA) was $20 / 30$ in the right eye and $20 / 40$ in the left eye. His manifest refraction in the right eye was $+2.75-1 \times 105$ with best-corrected visual acuity (BCVA) of 20/15-2; and +3 $-1.25 \times 80$ with BCVA of 20/20-1 in the left eye. Intraocular pressure was $10 \mathrm{~mm} \mathrm{Hg}$ in both eyes. The anterior segment and dilated eye exam were normal. Central ultrasonic pachymetry was 517 micron in the right eye and 518 micron in the left eye. Topography (NIDEK OPD) maps were normal in both eyes (Fig. 1).

The patient underwent uneventful Hyperopic LASIK, using the Hansatome microkeratome (Bausch and Lomb) with a $160 \mu \mathrm{m}$ plate and the NIDEK EC-5000 laser (NIDEK Inc. Fremont, California).

Three months postsurgery, his UCVA was 20/20 and 20/30-2 in the right and left eye respectively. Manifest refraction of $-0.5-0.5 \times 135$ in the right and $0.5-1 \times$ 100 in the left corrected to 20/20. In both eyes, the flaps were in good position, no wrinkles or folds.

The patient returned for general assessment 4 years later. He was very happy with his distance vision but noticed difficulty with reading. On examination, his UCVA was $20 / 20+3$ and $20 / 20-2$ in the right and left eye respectively. The manifest refraction was Plano $-0.25 \times 83$ in the right eye and -0.5 no cylinder in the left eye. He had traced nuclear 


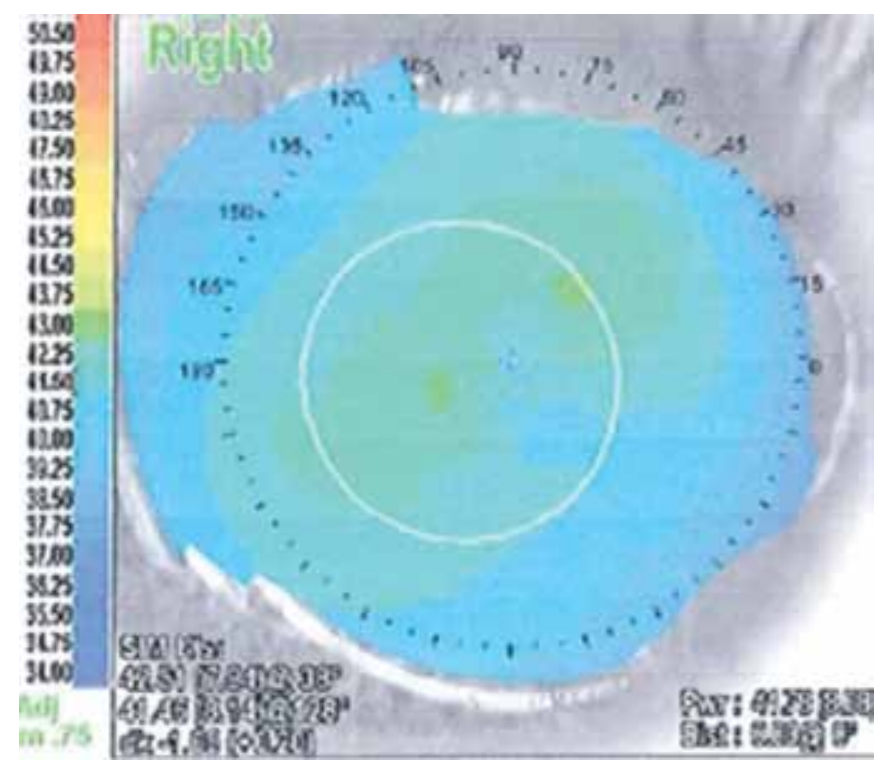

Fig. 1: Normal topography maps (NIDEK OPD) of the right eye taken before the LASIK procedure

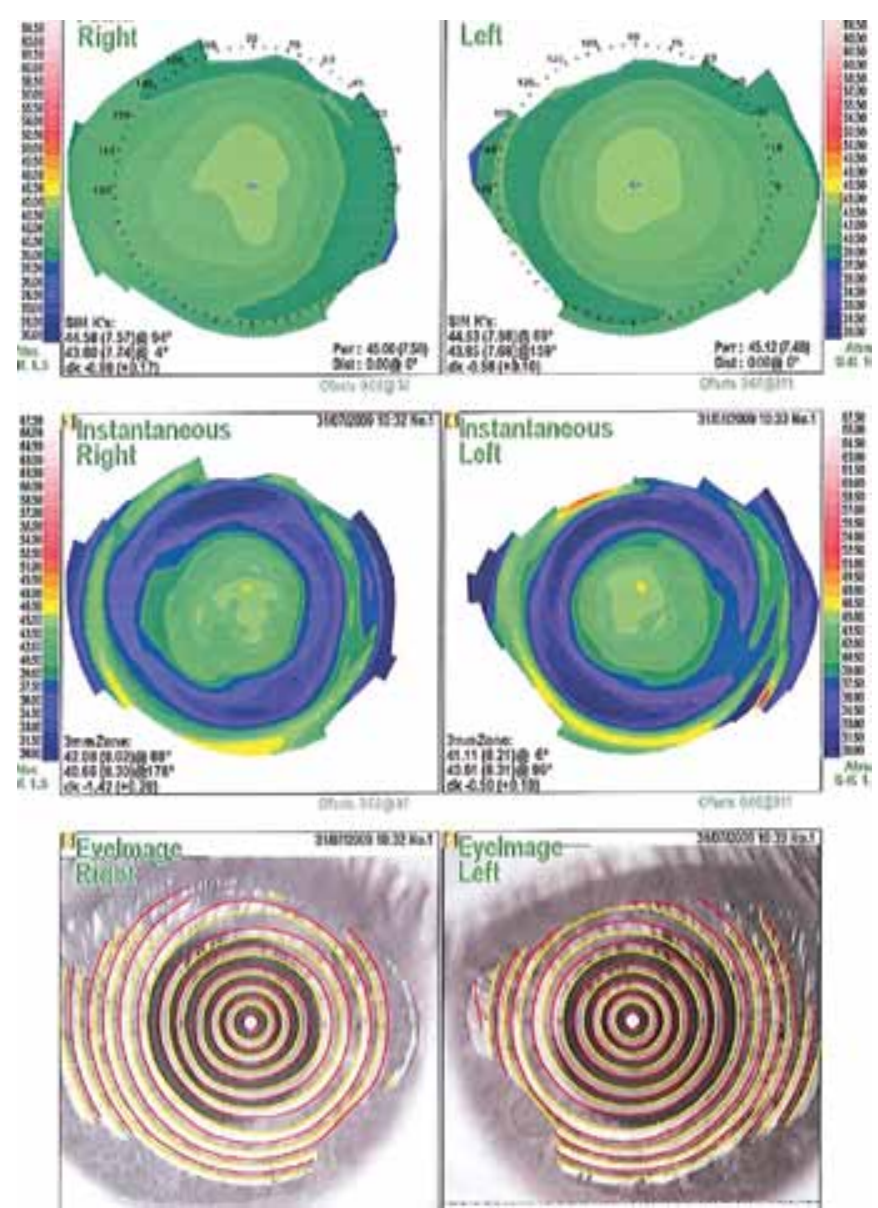

Fig. 2: Normal topography maps (NIDEK OPD) of the right and left eyes taken 4 years post LASIK procedure

sclerosis of the lens, fundus exam was normal. Ultrasound pachymetry was 501 micron in the right eye and 516 micron in the left. Topography (NIDEK OPD) maps of both eyes were normal (Fig. 2). He was prescribed reading glasses. The patient was doing well and was followed by his own eye care provider.

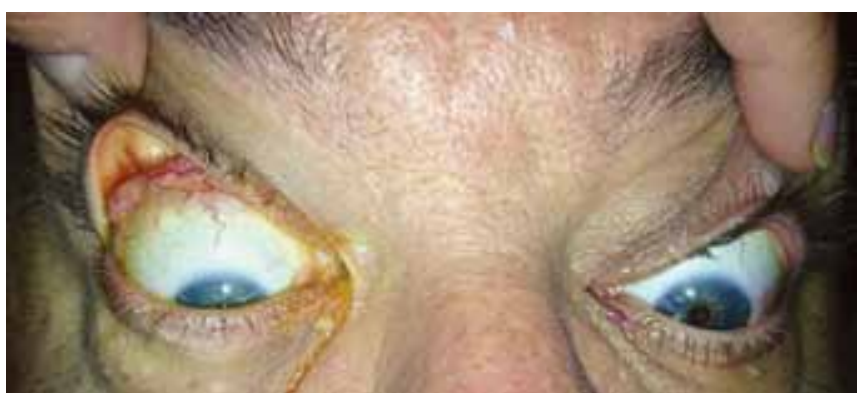

Fig. 3: Clinical picture of the patient floppy eyelids

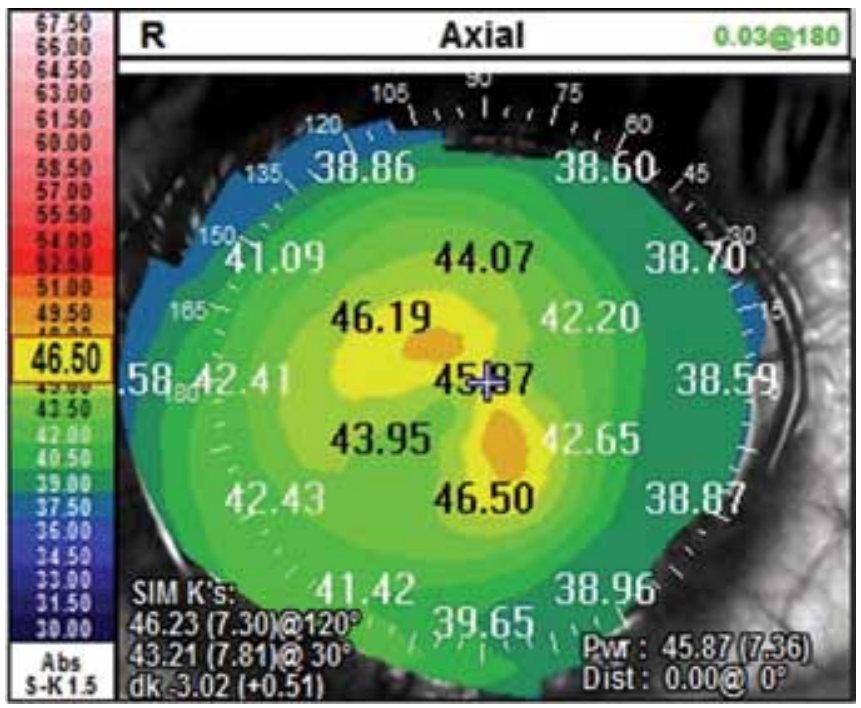

Fig. 4: Topography (NIDEK OPD) of the right eye taken 8 years post LASIK showing asymmetric bowtie pattern with skew deviation of the axis, corneal steepening, and high I-S difference

Eight years post LASIK, he was referred to our center again complaining of decreased distance vision in his right eye only, that was gradually progressing in the course of 6 months. The UCVA in the right eye was 20/60-2 with manifest refraction of $+1-2.25 \times 38$ best corrected to $20 / 25-2$. The left eye was doing well with UCVA $20 / 20-3$ corrected to $20 / 20+2$ with $+0.75-1.25$ $\times 102$. Examination of both eyelids revealed very elastic upper lids that became easily distorted and everted with minimal traction (Fig. 3). The flaps were in good position with no wrinkles or folds. There was bilateral minimal nuclear sclerosis of the lens and the dilated fundus exam was normal. Topography of the right eye (NIDEK OPD) revealed asymmetric bowtie and skew deviation of the axis. The cornea was steeper with Sim K's of 46.23@120 and 43.21@30. The inferior-superior (IS) difference was 2.43 (Fig. 4). On Oculus-Pentacam (Oculus Optikgerate GmbH, Wetzlar, Germany), map of the right eye shows inferior corneal steepening with posterior elevation of +34 (Fig. 5). On clinical examination, the patient was significantly overweight. He reported using a CPAP machine as he was diagnosed with sleep apnea 4 years prior to his visit. When specifically asked, he reported sleeping on his right side resting his face against his right arm. 


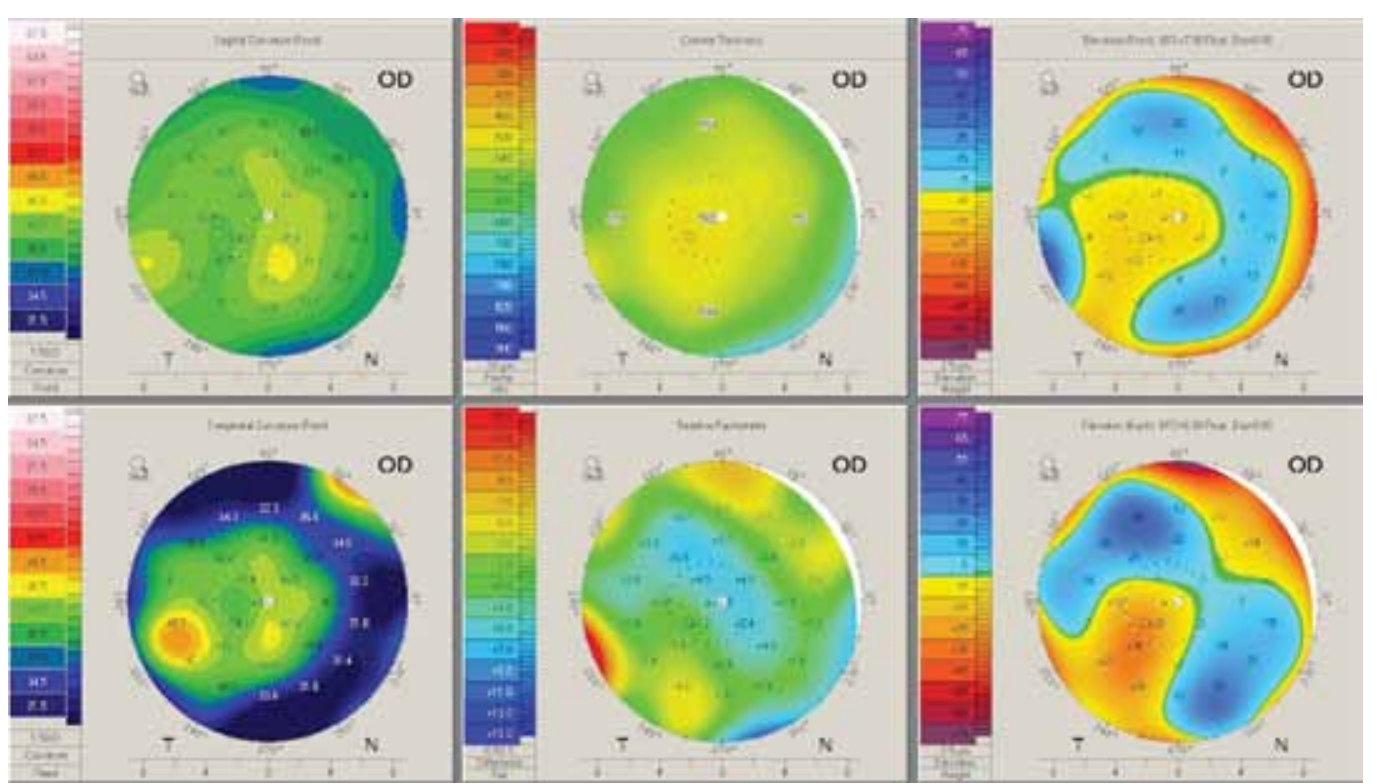

Fig. 5: Oculus-Pentacam map of the right eye shows inferior corneal steepening with posterior elevation of +34

The patient underwent corneal collagen cross-linking in his right eye and was advised to tape his eyelids when sleeping.

\section{DISCUSSION}

Post LASIK keratoectasia is a serious albeit rare complication. Known risk factors for ectasia include high myopia, undiagnosed forme fruste keratoconus, low residual stromal bed thickness, a large optical zone, and multiple enhancement procedures. ${ }^{12}$ In our report, the patient developed corneal ectasia 8 years following LASIK without any corneal risk factor. An important change in his general health was the new diagnosis of OSAS. The patient reported sleeping on the same side as the eye involved. In their study, Ezra et $\mathrm{al}^{13}$ described several mechanisms that have been proposed for this association of FES, OSAS, and keratoconus, including reperfusion ischemia ${ }^{5}$ and repeated mechanical trauma leading to matrix metalloproteinase expression and connective tissue remodeling. ${ }^{7}$ The mechanical stress theory is supported by the close association with keratoconus, a condition well described as being related to eye rubbing, which can lead to extracellular matrix ectasia. ${ }^{14}$

The association between OSAS and corneal ectasia is important and may provide awareness for the ophthalmologist to facilitate further management. This is important because, in addition to daytime somnolence, OSAS carries significant morbidity with a higher reported incidence of cardiovascular and cerebral sequelae. ${ }^{15}$

Our report emphasizes the importance of inquiring the patient about sleeping habits and a careful examination of eyelid laxity prior to and after refractive laser treatment. To our knowledge, this is the first documented case with topographic confirmation of post-LASIK ectasia developing after the diagnosis of OSAS.

\section{REFERENCES}

1. Culbertson WW, Ostler HB. The floppy eyelid syndrome. Am J Ophthalmol 1981;92:568-575.

2. Gonnering RS, Sonneland PR. Meibomian gland dysfunction in floppy eyelid syndrome. Ophthal Plast Reconstr Surg 1987; 3:99-103.

3. McNab AA. Floppy eyelid syndrome and obstructive sleep apnea. Ophthal Plast Reconstr Surg 1997;13:98-114.

4. Culbertson WW, Tseng SC. Corneal disorders in floppy eyelid syndrome. Cornea 1994;13:33-42.

5. Netland PA, Sugrue SP, Albert DM, et al. Histopathologic features of the floppy eyelid syndrome: involvement of tarsal elastin. Ophthalmology 1994;101:174-181.

6. Karesh JW, Nirankari VS, Hameroff SB. Eyelid imbrication: an unrecognized cause of chronic ocular irritation. Ophthalmology 1993;100:883-889.

7. Schlotzer-Schrehardt U, Stojkovic M, Hofmann-Rummelt C, et al. The pathogenesis of floppy eyelid syndrome: involvement of matrix metalloproteinases in elastic fiber degradation. Ophthalmology 2005;112:694-704.

8. Rossiter JD, Ellingham R, Hakin KN, et al. Corneal melt and perforation secondary to floppy eyelid syndrome in the presence of rheumatoid arthritis [letter]. Br J Ophthalmol 2002;86:48.

9. Donnenfeld ED, Perry HD, Gibralter RP, et al. Keratoconus associated with floppy eyelid syndrome. Ophthalmology 1991;98:1674-1678.

10. Langford JD, Linberg JV. A new physical finding in floppy eyelid syndrome. Ophthalmology 1998;105:165-169.

11. McNab AA. Reversal of floppy eyelid syndrome with treatment of obstructive sleep apnea. Clin Experiment Ophthalmol 2000; 28:125-126.

12. Randleman JB, et al. Risk factors and prognosis for corneal ectasia after LASIK. Ophthalmology 2003;110:p.267.

13. Ezra DG, Beaconsfield M, Sira M, et al. The Associations of Floppy Eyelid Syndrome: A Case Control Study. Ophthalmology 2010;117:831-838.

14. McMonnies CW. Abnormal rubbing and keratectasia. Eye Contact Lens 2007;33:265-271.

15. Ali LK, Avidan AY. Sleep-disordered breathing and stroke. Rev Neurol Dis 2008;5:191-198. 